\title{
QUEEN'S
UNIVERSITY
BELFAST
}

\section{Efficient Key Generation by Exploiting Randomness from Channel Responses of Individual OFDM Subcarriers}

Zhang, J., Marshall, A., Woods, R., \& Duong, T. Q. (2016). Efficient Key Generation by Exploiting Randomness from Channel Responses of Individual OFDM Subcarriers. IEEE Transactions on Communications, 64(6), 2578 2588. https://doi.org/10.1109/TCOMM.2016.2552165

Published in:

IEEE Transactions on Communications

Document Version:

Peer reviewed version

Queen's University Belfast - Research Portal:

Link to publication record in Queen's University Belfast Research Portal

Publisher rights

Copyright 2016 IEEE.

This work is made available online in accordance with the publisher's policies. Please refer to any applicable terms of use of the publisher.

\section{General rights}

Copyright for the publications made accessible via the Queen's University Belfast Research Portal is retained by the author(s) and / or other copyright owners and it is a condition of accessing these publications that users recognise and abide by the legal requirements associated with these rights.

Take down policy

The Research Portal is Queen's institutional repository that provides access to Queen's research output. Every effort has been made to ensure that content in the Research Portal does not infringe any person's rights, or applicable UK laws. If you discover content in the Research Portal that you believe breaches copyright or violates any law, please contact openaccess@qub.ac.uk. 


\title{
Efficient Key Generation by Exploiting Randomness from Channel Responses of Individual OFDM Subcarriers
}

\author{
Junqing Zhang, Alan Marshall, Senior Member, IEEE, Roger Woods, Senior Member, IEEE, and \\ Trung Q. Duong, Senior Member, IEEE
}

\begin{abstract}
Key generation from the randomness of wireless channels is a promising technique to establish a secret cryptographic key securely between legitimate users. This paper proposes a new approach to extract keys efficiently from channel responses of individual orthogonal frequency-division multiplexing (OFDM) subcarriers. The efficiency is achieved by (i) fully exploiting randomness from time and frequency domains and (ii) improving the cross-correlation of the channel measurements. Through the theoretical modelling of the time and frequency autocorrelation relationship of the OFDM subcarrier's channel responses, we can obtain the optimal probing rate and use multiple uncorrelated subcarriers as random sources. We also study the effects of non-simultaneous measurements and noise on the cross-correlation of the channel measurements. We find the cross-correlation is mainly impacted by noise effects in a slow fading channel and use a low pass filter (LPF) to reduce the key disagreement rate and extend the system's working signal-to-noise ratio range. The system is evaluated in terms of randomness, key generation rate, and key disagreement rate, verifying that it is feasible to extract randomness from both time and frequency domains of the OFDM subcarrier's channel responses.
\end{abstract}

Index Terms-Physical layer security, key generation, OFDM, time and frequency autocorrelation, channel reciprocity

\section{INTRODUCTION}

The broadcast nature of wireless communications allows all the users within range to hear the transmission, thus making it vulnerable to various active and passive attacks. Wireless network security and privacy thus has attracted many research interest [1]-[4]. In 5G networks, many new techniques have emerged, such as full-duplex communications [5], large-scale MIMO [6], etc. Physical layer security (PLS), which exploits channel characteristics to provide information-theoretic security for wireless communications, has been extensively

This work was supported by the Queen's University Belfast university studentship, Newton Institutional Links Grant 172719890, Royal Academy of Engineering Research Fellowship under Grant RF1415 \14\22, and USIreland R\&D Partnership USI033 'WiPhyLoc8' grant involving Rice University (USA), University College Dublin (Ireland) and Queen's University Belfast (Northern Ireland). This paper was presented in part at the IEEE GLOBECOM Workshop on Trusted Communications with Physical Layer Security (TCPLS), Austin, Texas, USA, Dec, 2014 and at the IEEE ICASSP, Brisbane, Australia, Apr, 2015.

J. Zhang, R. Woods and T. Q. Duong are with ECIT, Queen's University Belfast, Belfast, BT3 9DT, UK. (email: jzhang20@qub.ac.uk; r.woods@qub.ac.uk; trung.q.duong@qub.ac.uk)

A. Marshall is with Department of Electrical Engineering and Electronics, University of Liverpool, Liverpool, L69 3GJ, UK. (email: Alan.Marshall@liverpool.ac.uk) researched for the protection of future 5G networks [7]. Key generation, an active research direction of PLS, automatically generates keys at each side of two legitimate users, Alice and Bob, from the randomness of their common wireless channel [8], [9]. This technique exploits unpredictable channel characteristics, which is information theoretically secure [10]. It is low complexity and does not require the aid of other nodes, thus representing a promising alternative to public key cryptography to establish keys for classical symmetric encryption.

Key generation system is evaluated in terms of key randomness, key generation rate (KGR), and key disagreement rate (KDR). Randomness is the most important feature for the key sequence as the key generated is used for encryption and/or authentication. A less random key will result in a smaller search space by brute force attacks thus compromising the security of the cryptographic system. KGR is an essential factor for the practical application of key generation system. It quantifies the number of key bits generated in each second, which can be given as

$$
K G R=\frac{N_{k}}{T_{k}},
$$

where $N_{k}$ is the number of keys and $T_{k}$ is the time taken. Cryptography usually needs a key sequence with a certain length, e.g., advanced encryption standards (AES) requires a key length at least 128 bits, so a too low KGR will limit its application. KDR is the disagreement rate of the raw key bits quantized from the measurements, which is defined as

$$
K D R=\frac{\sum_{i=1}^{N_{k}}\left|K^{A}(i)-K^{B}(i)\right|}{N_{k}}
$$

where $K^{A}$ and $K^{B}$ are the keys generated at Alice and Bob, respectively. The disagreement is corrected by information reconciliation techniques. A lower KDR can always decrease the reconciliation overhead and reveal less information during the public discussion. Therefore, an efficient key generation system should have a high KGR and small KDR with the premise of generating random keys. KGR can be improved by leveraging fine-grained channel state information (CSI) and exploiting randomness fully from temporal, frequency, and spatial domains. KDR can be decreased by improving the signal cross-correlation. In the following of this introduction, we review key generation channel parameters, randomness 
exploitation from different domains, and measurement crosscorrelation improvement.

Several practical and simulation systems have been reported for extracting keys from coarse-grained channel parameters, such as received signal strength (RSS) [11]-[18], channel phase in narrowband systems [19], [20], and deep fades of the signal envelop [21], etc. However, all of this work only extracts keys from a single dimension or a single frequency, which results in a low KGR and therefore limits their practical application. Although some research effort has attempted to improve the KGR by leveraging multi-antenna [14] and/or multibit quantization [16], it remains that these single-dimensional approaches lose much useful information.

Key generation from fine-grained CSI can achieve a higher KGR [22], [23]. A practical CSI-based key generation system was proposed to quantize channel responses in the frequency domain from all subcarriers in orthogonal frequencydivision multiplexing (OFDM) systems [22], which may introduce redundancy and correlation between keys especially in a frequency flat fading channel. Later, another CSI-based key generation protocol called KEEP that uses a validationrecombination mechanism was designed [23]. However, it is difficult to reach an agreement in low signal-to-noise ratio (SNR) environments as even a single bit mismatch will result in a failure of the entire process and thereafter require a new validation-recombination process. In this paper, we also exploit channel randomness from CSI but in a different manner, i.e., by extracting keys from the channel responses of individual OFDM subcarriers over time. This provides a thorough theoretical modelling of the system and channel, and enables us to obtain the optimal probing rate and maximize the KGR, which will be discussed later.

The randomness in time, frequency, and spatial domains can be used for key extraction. While spatial randomness exploitation has been extensively analyzed in [24]-[28], this paper focuses on randomness extraction from time and frequency domains. The temporal randomness is the main random source for key generation as it can be easily introduced by the movement of the users and/or any objects within the communication environments [11]-[16], [25]. Frequency variation is another random source which currently receives less attention. Frequency diversity is intrinsically determined by the delay spread of multipath channel, which has been used for key generation in ultrawideband channel [29]. There has been research reported exploiting frequency diversity from RSS using channel hopping [17], [18], from channel measurements of multiple FM radios [30], or from CSI in IEEE 802.11 OFDM systems [22], [23]. However, a detailed theoretical modelling and analysis of the temporal and frequency correlation is missing, which restricts the capability to exploit the randomness of the channel.

The cross-correlation of the channel measurements of Alice and Bob is essential for the success of key generation. The statistical features of the same carrier frequency at each end are reciprocal, which is the basis of this type of key generation [13]. Most of the current commercial devices work in half-duplex mode, and the cross-correlation of the received signals measured at Alice and Bob are impacted by the non- simultaneous measurements (probing) and noise. Even when Alice and Bob measure the channel at the same frequency and time using full-duplex hardware ${ }^{1}$, the noise at each side will still be independent and uncorrelated as they reside in two different hardware platforms. Non-identical channel measurements introduce key disagreement, while a too high KDR may result in a failure of the entire key generation process. There has been research in compensating the nonsimultaneous measurements using interpolation [15], [16] and suppressing the noise by filtering [11], [21], [22], [31]. However, the cross-correlation of the channel measurements has not yet been modelled theoretically, therefore, the design of the interpolation or filter algorithms are mainly empirical, resulting in a less effective improvement on the correlation.

In this paper, we propose a new efficient CSI-based key generation system by exploiting both the temporal and frequency randomness from channel responses of individual OFDM subcarriers. As part of the ongoing WiPhyLoc8 project [32], this paper aims to develop novel and practical approaches for wireless security. We carried out the analysis by considering a practical scenario, i.e., by incorporating an IEEE 802.11 OFDM transceiver model and a time-varying multipath channel model. This offers guidelines to implement a real key generation system in the testbed. Our contributions are:

- Efficient key generation from the channel responses of individual OFDM sucbarriers. By theoretically modelling the subcarrier's channel responses, it is demonstrated that they are fine-grained channel parameter which provides detailed channel properties in both time and frequency domains.

- By theoretically modelling the time and frequency autocorrelation relationship of OFDM subcarrier's channel responses, we can fully exploit the randomness of the channel in both time and frequency domains by obtaining the optimal probing rate and using multiple subcarriers as random source. Therefore, we can greatly improve the KGR while guaranteeing the randomness of the keys.

- By theoretically modelling the effects of nonsimultaneous measurements and noise on the crosscorrelation of the channel measurements, we found that noise plays a more dominant role in a slow fading channel and thereof employed a finite impulse response (FIR) low pass filter (LPF) to effectively target the high frequency components of the noise and significantly improve the correlation. The employment of LPF helps reduce the KDR and extend the working SNR range.

In previous work, we have analyzed the temporal variation of the OFDM subcarrier's channel response and verified its application in key generation in [33] and used an LPF to improve the correlation of the measurements in [34]. In this paper, we considerably extend and complement this work by providing a theoretical and extensive modelling and analysis of the channel, time and frequency domains autocorrelation relationship, and cross-correlation of the channel measurements.

\footnotetext{
${ }^{1}$ In full-duplex system, transceivers can work in different carrier frequencies, but their channel responses will be different and cannot be used for key generation.
} 
The rest of the paper is organized as follows. Section II describes OFDM channel model and the simulation model. Sections III and IV theoretically analyze the time and frequency autocorrelation relationship of the channel responses and channel measurements cross-correlation, respectively. The performance of our key generation system is evaluated in Section V. Section VI concludes the paper.

\section{Channel Model}

\section{A. Multipath Channel}

A dynamic multipath channel with lots of reflection, scattering, and refraction of the electromagnetic wave is an ideal random source for key generation. The channel impulse response (CIR) $h(\tau, t)$ of such a multipath channel can be written as

$$
h(\tau, t)=\sum_{l=0}^{L-1} h\left(\tau_{l}, t\right) \delta\left(\tau-\tau_{l}\right),
$$

where $h\left(\tau_{l}, t\right)$ and $\tau_{l}$ are the attenuation and delay of $l^{t h}$ channel tap, respectively, $\tau_{l}=l T$, and $T$ is the sampling period of the system, $L$ is the total number of the channel taps and $\delta(\cdot)$ is the Dirac delta function.

When there is rich scattering, the channel can be modelled as a wide sense stationary uncorrelated scattering (WSSUS) random process [35]. Under this model, the attenuation of each channel tap $h\left(\tau_{l}, t\right)$ is a WSS random process and the attenuations of any two taps with different delays, i.e., $h\left(\tau_{l}, t\right)$ and $h\left(\tau_{j}, t\right)$, are uncorrelated. Therefore, the temporal autocorrelation function (ACF) $r_{h}(\tau, \Delta t)$ is given by

$$
r_{h}(\tau, \Delta t)=E\left\{h(\tau, t)^{*} h(\tau, t+\Delta t)\right\} .
$$

The normalized temporal ACF of $h\left(\tau_{l}, t\right)$ can also be further defined as

$$
R_{h}\left(\tau_{l}, \Delta t\right)=\frac{r_{h}\left(\tau_{l}, \Delta t\right)}{r_{h}\left(\tau_{l}, 0\right)} .
$$

WSS is a common channel model and has been verified by experimental measurements for a rich scattering environment [36]. It is suitable to describe the channel correlation when the Doppler spread is fixed, i.e., the channel is always changing in the same rate. In real channels, this assumption may not be satisfied due to the uncontrolled movement of objects and thereof variable Doppler spread. Under this circumstance, the channel can be divided into small time frames and each frame can be approximated as a WSS random process [37].

\section{B. OFDM Model}

In OFDM systems, the transmitted signal consists of multiple OFDM symbols $x_{q}[m]$, which can be written as

$$
x_{q}[m]=\frac{1}{\sqrt{M}} \sum_{k=0}^{M-1} X_{q}[k] e^{j 2 \pi k m / M},
$$

where $X_{q}[k]$ is the data modulated to the $k^{t h}$ subcarrier in $q^{t h}$ OFDM symbol in frequency domain at $t_{q}, x_{q}[m]$ is the $m^{\text {th }}$ sample in $q^{\text {th }}$ OFDM symbol in time domain, and $M$ is the number of total subcarriers. In an OFDM system with
$B W \mathrm{~Hz}$ channel spacing and $M$ evenly distributed subcarriers, the frequency of each subcarrier is given as

$$
f_{k}=k \frac{B W}{M},
$$

where $B W=\frac{1}{T}$.

The transmitted signal $x_{q}[m]$ experiences the multipath effect and is affected by the noise. After synchronization, the received signal can be written as [38]

$$
y_{q}[m]=\sum_{l=0}^{L-1} x_{q}\left[m-\varepsilon_{q}-l\right] h_{q}[l]+n_{q}[m],
$$

where $n_{q}[m]$ is the additive Gaussian white noise (AWGN) and $n_{q}[m] \sim C \mathcal{N}\left(0, \sigma_{n}^{2}\right) ; \varepsilon_{q}$ is the time offset due to the imperfect synchronization and is determined by the synchronization algorithm, SNR, and the multipath effect; $h_{q}[l]$ is the discrete form of $h\left(\tau_{l}, t\right)$, and is assumed to remain unchanged during one OFDM symbol, which is a fair assumption in a slow fading environment.

When the synchronization time offset is small, the equivalent frequency domain value $Y_{q}[k]$ can be written as [38]

$$
\begin{aligned}
Y_{q}[k] & =\frac{1}{\sqrt{M}} \sum_{m=0}^{M-1} y_{q}[m] e^{-j 2 \pi k m / M} \\
& =X_{q}[k] H_{q}[k] e^{-j 2 \pi k \varepsilon_{q} / M}+w_{q}[k],
\end{aligned}
$$

where

$$
\begin{aligned}
& H_{q}[k]=\sum_{l=0}^{L-1} h_{q}[l] e^{-j 2 \pi k l / M} ; \\
& w_{q}[k]=\frac{1}{\sqrt{M}} \sum_{m=0}^{M-1} n_{q}[m] e^{-j 2 \pi k m / M} .
\end{aligned}
$$

Least square (LS) channel estimation can get a noisy observation of the channel responses in OFDM systems, which can be given as

$$
\widehat{H}_{q}[k]=\frac{Y_{q}[k]}{X_{q}[k]}=\widetilde{H}_{q}[k]+\widehat{w}_{q}[k],
$$

where

$$
\begin{aligned}
\widetilde{H}_{q}[k] & =H_{q}[k] e^{-j 2 \pi k \varepsilon_{q} / M} ; \\
\widehat{w}_{q}[k] & =\frac{w_{q}[k]}{X_{q}[k]} .
\end{aligned}
$$

It can be calculated that

$$
\begin{aligned}
\sigma_{\widetilde{H}}^{2} & =\sigma_{H}^{2}=\sum_{l=0}^{L-1} \sigma_{h_{l}}^{2} ; \\
\sigma_{\widehat{w}}^{2} & =\sigma_{w}^{2}=\sigma_{n}^{2} ; \\
\sigma_{\overparen{H}}^{2} & =\sigma_{H}^{2}+\sigma_{w}^{2} .
\end{aligned}
$$

The above variances do not depend on the subcarrier index $k$ which is omitted. Therefore, the channel responses of all the subcarriers will have the same SNR which can be given as

$$
S N R_{f}=\frac{E\left[\left|\widetilde{H}_{q}[k]\right|^{2}\right]}{E\left[|\widehat{w}[k]|^{2}\right]}=\frac{\sigma_{H}^{2}}{\sigma_{w}^{2}} .
$$


TABLE I

SIMULATION PARAMETERS

\begin{tabular}{|l|l|}
\hline IEEE 802.11 OFDM channel spacing $B W$ & $20 \mathrm{MHz}$ \\
\hline Hardware sampling frequency $\frac{1}{T}$ & $20 \mathrm{MHz}$ \\
\hline Doppler spread $f_{d}$ & $6 \mathrm{~Hz}$ \\
\hline Root mean square delay spread $\sigma_{\tau}$ & $50 \mathrm{~ns}$ \\
\hline Total sampling time & $500 \mathrm{~s}$ \\
\hline
\end{tabular}

It should be noted that as the mean square error (MSE) of LS channel estimation is inversely proportional to SNR [39], which is not as accurate as some other algorithms, e.g., minimum mean square error (MMSE) channel estimation. However, it is widely applied in commercial OFDM systems such as IEEE 802.11 OFDM. Therefore, in order to make the analysis in this paper more general, LS channel estimation is still adopted.

\section{Simulation Model}

A Matlab simulation model is implemented as an example for analysis. The transceiver is implemented according to the IEEE 802.11 OFDM protocol [40]. The statistical channel is modelled as a time-variant multipath fading channel [41] and a WSSUS random process. The average power of each channel tap follows an exponential-decay power delay profile and a Bell-shaped Doppler power spectrum [42], which is recommended by the IEEE working group. The normalized Doppler power spectral density (PSD) can be given as

$$
S(f)=\frac{\sqrt{A} /\left(\pi f_{d}\right)}{1+A\left(\frac{f}{f_{d}}\right)^{2}},
$$

where $A$ is a constant, e.g., in IEEE 802.11 channel, $A=9$ and $f_{d}$ is the Doppler spread, whose values were found to be up to approximately $6 \mathrm{~Hz}$ at a center frequency of $5.25 \mathrm{GHz}$ and up to approximately $3 \mathrm{~Hz}$ at a center frequency of $2.4 \mathrm{GHz}$ by experiments in indoor environment [42]. PSD and normalized temporal ACF form an IFFT pair. Therefore, the corresponding temporal ACF of the Bell-shaped Doppler spectrum can be given by

$$
R(\Delta t)=e^{-\frac{2 \pi f_{d}}{\sqrt{A}} \Delta t} .
$$

For the simplicity of analysis, all the channel taps are modelled to have the same PSD.

The simulation parameters are shown in Table I. Unless otherwise specified, the results in this paper are based on the above simulation model and parameters. However, it is worth noting that our system and analyses work for other OFDM standards and multipath channels as well.

\section{AnAlysis of Time AND FREQUency AUTOCORRELATION}

In a dynamic multipath environment, the signal experiences time-selective and frequency-selective fading. In order to generate a random key sequence, the sampled data should be uncorrelated. The correlation relationship of $H_{q}[k]$ can be characterized by the time and frequency ACF and given as [43]

$$
\begin{aligned}
r_{H}(\Delta f, \Delta t) & =E\left\{H_{q}[k]^{*} H_{p}[i]\right\} \\
& =\sum_{l=0}^{L-1} r_{h}\left(\tau_{l}, \Delta t\right) e^{-j 2 \pi \Delta f \tau_{l}},
\end{aligned}
$$

and the normalized correlation function of $H_{q}[k]$ can be written as

$$
\begin{aligned}
R_{H}(\Delta f, \Delta t) & =\frac{r_{H}(\Delta f, \Delta t)}{r_{H}(0,0)} \\
& =\frac{\sum_{l=0}^{L-1} r_{h}\left(\tau_{l}, \Delta t\right) e^{-j 2 \pi \Delta f \tau_{l}}}{\sum_{l=0}^{L-1} r_{h}\left(\tau_{l}, 0\right)},
\end{aligned}
$$

where $\Delta f=f_{i}-f_{k}=(i-k) \frac{B W}{M}, \Delta t=t_{p}-t_{q}$.

The time and frequency ACF of $\widetilde{H}_{q}[k]$ and $\widehat{w}_{q}[k]$ can be calculated as

$$
r_{\widetilde{H}}\left(f_{k}, f_{i}, \Delta t\right)=r_{H}(\Delta f, \Delta t) E\left\{e^{j 2 \pi\left(k \varepsilon_{q}-i \varepsilon_{p}\right) / M}\right\},
$$

and

$$
r_{\widehat{w}}(\Delta f, \Delta t)=r_{w}(\Delta f, \Delta t)=\delta(\Delta f) \delta(\Delta t) \sigma_{w}^{2},
$$

respectively.

Therefore, the time and frequency ACF of the channel estimation $\widehat{H}_{q}[k]$ can be given as

$$
\begin{aligned}
& r_{\widehat{H}}\left(f_{k}, f_{i}, \Delta t\right)=r_{\widetilde{H}}\left(f_{k}, f_{i}, \Delta t\right)+r_{\widehat{w}}(\Delta f, \Delta t) \\
& =r_{H}(\Delta f, \Delta t) E\left\{e^{j 2 \pi\left(k \varepsilon_{q}-i \varepsilon_{p}\right) / M}\right\}+r_{w}(\Delta f, \Delta t),
\end{aligned}
$$

and the normalized correlation function of $\widehat{H}_{q}[k]$ can be written as

$$
\begin{aligned}
& R_{\widehat{H}}\left(f_{k}, f_{i}, \Delta t\right)=\frac{r_{\widehat{H}}\left(f_{k}, f_{i}, \Delta t\right)}{r_{\widehat{H}}\left(f_{k}, f_{k}, 0\right)} \\
& =\frac{r_{H}(\Delta f, \Delta t) E\left\{e^{j 2 \pi\left(k \varepsilon_{q}-i \varepsilon_{p}\right) / M}\right\}+r_{w}(\Delta f, \Delta t)}{r_{H}(0,0)+r_{w}(0,0)} \\
& =\frac{R_{H}(\Delta f, \Delta t) S N R_{f} E\left\{e^{j 2 \pi\left(k \varepsilon_{q}-i \varepsilon_{p}\right) / M}\right\}+\delta(\Delta f) \delta(\Delta t)}{1+S N R_{f}} .
\end{aligned}
$$

\section{A. Time Correlation}

In a dynamic environment with random movement, the signal experiences time-selective fading, which is the main random source for key generation. The users harvest the entropy by probing the channel and getting the channel measurements. A smaller probing rate enjoys a higher KGR but compromises the randomness of the generated key sequence due to the correlation between the sampled data, while a larger probing rate results in a lower KGR and limits its practical application. Key sequence is used in cryptographic applications and should be random. Optimal probing rate is defined as the minimum probing rate which can guarantee the randomness of the key sequence.

The channel variation in the time domain can be characterized by the temporal ACF. The channel coherence time can statistically approximate the time duration over which the CIR is essentially invariant and quantifies the similarity of the channel response [44]. It is usually defined by the time over 
which the coefficient of the temporal ACF is above $50 \%$. The definition can be further extended to $X \%$ coherence time [45] and be used for all the random process, which is given as

$$
R\left(T_{c}(X \%)\right)=X \% .
$$

In this section, under the assumption that $h(\tau, t)$ is a WSSUS random process, we model the $R_{\widehat{H}}\left(f_{k}, \Delta t\right)$ and $R_{H}\left(f_{k}, \Delta t\right)$, and prove $\widehat{H}_{q}[k]$ and $H_{q}[k]$ are also WSS random processes. The WSS property guarantees that the data sampled by the same time interval $\Delta t$ will have the same correlation relationship. Based on the temporal ACF, the optimal probing rate can be determined.

For the $k^{t h}$ subcarrier, the mean value of $H_{q}[k]$ is 0 . The normalized temporal ACF of the $H_{q}[k]$ can be obtained by letting $\Delta f=0$ in (22), which can be given as

$$
\begin{aligned}
R_{H}(0, \Delta t) & =\frac{\sum_{l=0}^{L-1} r_{h}\left(\tau_{l}, \Delta t\right)}{\sum_{l=0}^{L-1} r_{h}\left(\tau_{l}, 0\right)} \\
& =\frac{\sum_{l=0}^{L-1}\left(r_{h}\left(\tau_{l}, 0\right) \times R_{h}(\Delta t)\right)}{\sum_{l=0}^{L-1} r_{h}\left(\tau_{l}, 0\right)} \\
& =R_{h}(\Delta t) .
\end{aligned}
$$

The second equality holds because in this paper, all the channel taps have the same temporal $\mathrm{ACF}$, i.e.,

$$
R_{h}\left(\tau_{l}, \Delta t\right)=R_{h}(\Delta t), l=0,1, \ldots, L-1 .
$$

As the mean value is a constant and ACF only depends on the time delay, $H_{q}[k]$ is a WSS random process.

The normalized temporal ACF of the imperfectly synchronized channel estimation can be calculated by letting $f_{i}=f_{k}$, i.e, $\Delta f=0$, in (26) and written as

$R_{\widehat{H}}\left(f_{k}, \Delta t\right)=\frac{R_{H}(0, \Delta t) S N R_{f} E\left\{e^{j 2 \pi k\left(\varepsilon_{q}-\varepsilon_{p}\right) / M}\right\}+\delta(\Delta t)}{1+S N R_{f}}$.

$R_{\widehat{H}}\left(f_{k}, \Delta t\right)$ is also only determined by $\Delta t$ and irrelevant to the observation time, therefore, it is a WSS process.

Fig. 1 shows several results for $R_{h}\left(\tau_{l}, \Delta t\right), R_{H}\left(f_{k}, \Delta t\right)$, and $R_{\widehat{H}}\left(f_{k}, \Delta t\right)$ from the simulation to validate the above analytic analysis. $R_{\widehat{H}}^{\prime}\left(f_{1}, \Delta t\right)$ is calculated by letting $\varepsilon_{q}-\varepsilon_{p}=0$ to show the reference ACF if perfect synchronization were achieved. Firstly, all the shown $R_{h}\left(\tau_{l}, \Delta t\right)$ and $R_{H}\left(f_{k}, \Delta t\right)$ values are equal, which matches the analytic expression (28). Secondly, $R_{H}\left(f_{k}, \Delta t\right)$ and $R_{\widehat{H}}\left(f_{k}, \Delta t\right)$ observed at $t_{1}$, match their counterparts at $t_{2}$ quite well, respectively, which validates that $H_{q}[k]$ and $\widehat{H}_{q}[k]$ are WSS random processes. Lastly, $R_{\widehat{H}}\left(f_{k}, \Delta t\right)$ observed at $t_{1}$ vary according to the subcarrier index $k$, which matches the analytical expression (30).

Previous key generation research has claimed that the probing rate should be larger than $50 \%$ coherence time in order to get a random key sequence. However, it has been observed that whenever the experiments were carried out, the authors usually chose the probing rate to be large enough to exceed the expected coherence time [22]. However, in this paper we calculate the $X \%$ coherence time $T_{c}(X \%)$ based on the temporal ACF of the random process, and use it as the probing rate to sample the channel. The optimal probing

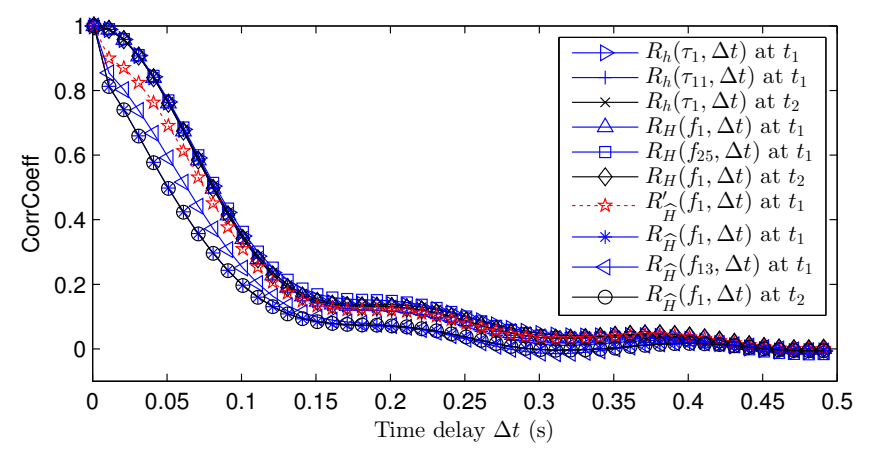

Fig. 1. Temporal ACFs. $S N R=10 \mathrm{~dB} . R_{h}\left(\tau_{l}, \Delta t\right), R_{H}\left(f_{k}, \Delta t\right)$ and $R_{\widehat{H}}\left(f_{k}, \Delta t\right)$ observed at $t_{1}$ and $t_{2} \cdot t_{2}=t_{1}+10 \mathrm{~s}$

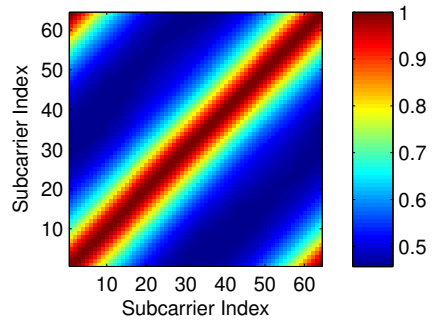

(a)

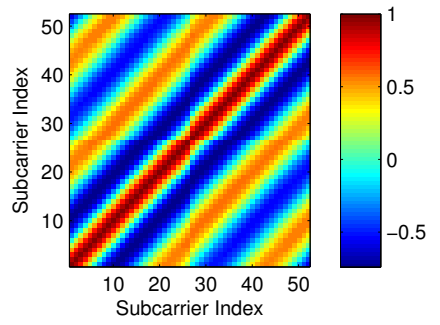

(b)
Fig. 2. Frequency ACFs, $\sigma_{\tau}=50 \mathrm{~ns}, S N R=10 \mathrm{~dB}$. (a) $R_{H}(\Delta f, 0)$; (b) $R_{\widehat{H}}\left(f_{k}, f_{i}, 0\right)$.

rate can then be found by evaluating the randomness of the key sequence sampled by different $T_{c}(X \%)$, which is a major difference from previous work. The detailed results for this procedure are presented in Section V-A1.

\section{B. Frequency Correlation}

In a multipath environment with rich scattering, the signal experiences frequency-selective fading, which is another valid random source that can be used for key generation. However, there will be correlation between adjacent frequencies. In this section, we exploit the frequency correlation relationship of the channel estimation $\widehat{H}_{q}[k]$.

The normalized frequency ACFs of $H_{q}[k]$ and $\widehat{H}_{q}[k]$ can be obtained by letting $\Delta t=0$ in (22) and (26) and are written as

$$
R_{H}(\Delta f, 0)=\frac{r_{H}(\Delta f, 0)}{r_{H}(0,0)}
$$

and

$R_{\widehat{H}}\left(f_{k}, f_{i}, 0\right)=\frac{R_{H}(\Delta f, 0) S N R_{f} E\left\{e^{j 2 \pi(k-i) \varepsilon_{q} / M}\right\}+\delta(\Delta f)}{1+S N R_{f}}$,

respectively, and shown in Fig. 2.

The frequency ACFs of $\widehat{H}_{q}[k]$ indicates that it is feasible to extract keys from multiple subcarriers that are separated by a certain frequency. This is verified by the randomness test and the detailed results are shown in Section V-A2. 


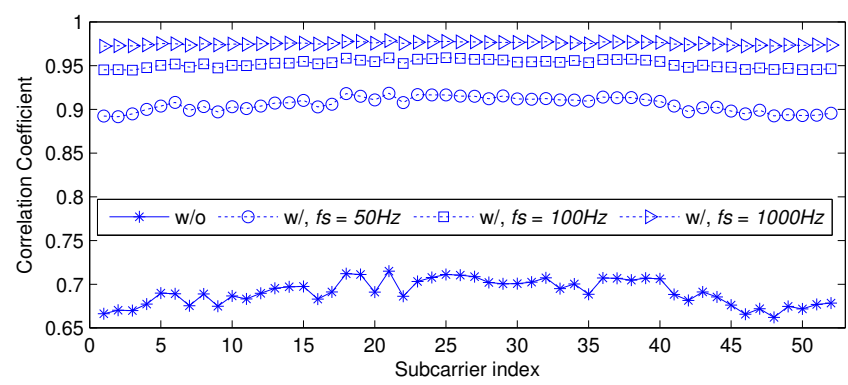

Fig. 3. $\rho\left(\widehat{H}_{t_{A}}^{A}[k], \widehat{H}_{t_{B}}^{B}[k]\right)$ of all the subcarriers. $S N R=6 \mathrm{~dB}$.

\section{Analysis AND IMPROVEMENT OF MEASUREMENTS CROSS-CORRELATION}

In this section, we analyze the effects of non-simultaneous measurements and noise on the signal cross-correlation and improve the correlation by an FIR LPF. We consider halfduplex hardware to make our analysis more general. The estimated channel responses of Alice and Bob can be given as

$$
\begin{aligned}
\widehat{H}_{t_{A}}^{A}[k] & =\widetilde{H}_{t_{A}}[k]+\widehat{w}_{t_{A}}^{A}[k] ; \\
\widehat{H}_{t_{B}}^{B}[k] & =\widetilde{H}_{t_{B}}[k]+\widehat{w}_{t_{B}}^{B}[k],
\end{aligned}
$$

where $t_{A}$ and $t_{B}$ are the measurement time of Alice and Bob, respectively. The value $\Delta t_{A B}=\left|t_{A}-t_{B}\right|$ is deliberately kept as small as possible to ensure that $\widetilde{H}_{t_{A}}^{A}[k]$ and $\widetilde{H}_{t_{B}}^{B}[k]$ are highly correlated in a slow fading channel. The noises $\widehat{w}_{t_{A}}^{A}[k]$ and $\widehat{w}_{t_{B}}^{B}[k]$ reside in two hardware platforms and therefore are independent.

\section{A. Cross-Correlation Relationship}

Cross-correlation relationship describes the similarity between the measured channel responses of Alice and Bob. The covariance between $\widehat{H}_{t_{A}}^{A}[k]$ and $\widehat{H}_{t_{B}}^{B}[k]$ can be calculated as

$$
\operatorname{cov}\left(\widehat{H}_{t_{A}}^{A}[k], \widehat{H}_{t_{B}}^{B}[k]\right)=\operatorname{cov}\left(H_{t_{A}}[k], H_{t_{B}}[k]\right) E\left\{e^{j 2 \pi \Delta \varepsilon^{\prime} k / M}\right\},
$$

where $\Delta \varepsilon^{\prime}=\varepsilon_{t_{A}}-\varepsilon_{t_{B}}$.

The correlation coefficient between $\widehat{H}_{t_{A}}^{A}[k]$ and $\widehat{H}_{t_{B}}^{B}[k]$ can be given as

$$
\rho\left(\widehat{H}_{t_{A}}^{A}[k], \widehat{H}_{t_{B}}^{B}[k]\right)=\frac{\operatorname{cov}\left(H_{t_{A}}[k], H_{t_{B}}[k]\right) E\left\{e^{j 2 \pi \Delta \varepsilon^{\prime} k / M}\right\}}{\sigma_{H}^{2}+\sigma_{w}^{2}},
$$

and the average correlation coefficient of all the subcarriers can be calculated by

$$
\bar{\rho}=\frac{1}{M} \sum_{k=0}^{M-1} \rho\left(\widehat{H}_{t_{A}}^{A}[k], \widehat{H}_{t_{B}}^{B}[k]\right) .
$$

The cross-correlation coefficients of all the subcarriers are shown in Fig. 3 using SNR $=6 \mathrm{~dB}$ as an example. It may be observed that the cross-correlation coefficients are slightly different due to the imperfect synchronization at the receiver.

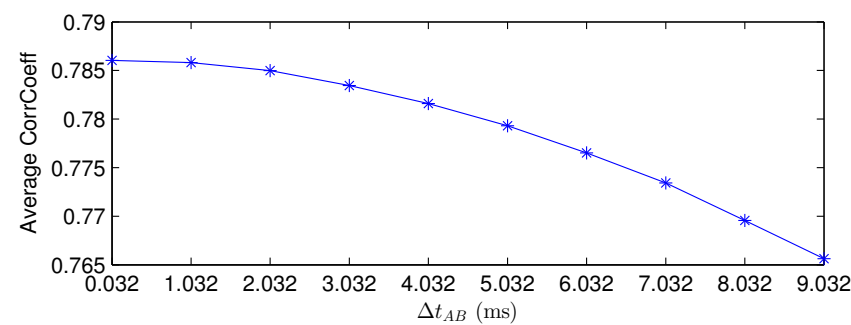

Fig. 4. The average correlation coefficient against $\Delta t_{A B} . S N R=10 \mathrm{~dB}$.

1) Effect of Non-Simultaneous Measurements: Although Alice and Bob do not measure the channel at the same time, the channel does not change much in a slow fading environment as long as $\Delta t_{A B}$ is small enough. The average correlation coefficient against $\Delta t_{A B}$ is shown in Fig. 4.

As may be observed from the figure, $\Delta t_{A B}$ does not affect the average correlation coefficients much when it is small. This time resolution is easy to satisfy. For example, in a $20 \mathrm{MHz}$ channel spacing IEEE 802.11 OFDM system, the sampling time difference between Alice and Bob can be configured in the order of $0.1 \mathrm{~ms}$.

2) Effect of Noise: Noise is then the main factor that impacts the measurements. The frequency domain components of the $H_{t}[k]$ and $\widehat{H}_{t}[k]$ are shown in Fig. 5 (a) and Fig. 5 (b), respectively. As shown in (28), $H_{t}[k]$ has the same temporal ACF as the channel taps, therefore, their PSD $S(f)$ are the same as well. The main energy of $H_{t}[k]$ is then concentrated in $\left[0, f_{d}\right]$. This can also be observed from Fig. 5 (a). Therefore, an LPF can be designed to eliminate the high frequency components which flood $\widehat{H}_{t}[k]$.

3) Correlation Relationship Approximation: As the channel does not change much during $\Delta t_{A B}$, the correlation coefficient can be approximated to

$$
\begin{aligned}
\rho\left(\widehat{H}_{t_{A}}^{A}[k], \widehat{H}_{t_{B}}^{B}[k]\right) & \approx \frac{\operatorname{cov}\left(H_{t_{A}}[k], H_{t_{A}}[k]\right) E\left\{e^{j 2 \pi \Delta \varepsilon^{\prime} k / M}\right\}}{\sigma_{H}^{2}+\sigma_{w}^{2}} \\
& =\frac{\sigma_{H}^{2} E\left\{e^{j 2 \pi \Delta \varepsilon^{\prime} k / M}\right\}}{\sigma_{H}^{2}+\sigma_{w}^{2}} \\
& =\frac{S N R_{f}}{1+S N R_{f}} E\left\{e^{j 2 \pi \Delta \varepsilon^{\prime} k / M}\right\}
\end{aligned}
$$

The cross-correlation coefficients are mainly determined by the SNR. We calculate the average correlation coefficients of all the subcarriers against SNR and show the results in Fig. 6. The theoretical curve is calculated by the analytical expression (38) which assumes perfect synchronization, i.e., $\Delta \varepsilon^{\prime}=0$. As may be observed from the figure, when SNR is low, the correlation coefficients exhibit large deviations from the theoretical ones. This is because in low SNR environments, there is a greater difference in the time offsets of Alice and Bob.

\section{B. Measurements Correlation Improvement}

An FIR LPF is proposed to effectively target the elimination of the noise and improve the SNR and correlation relationship. The parameters of the LPF are shown in Table II. As the main 

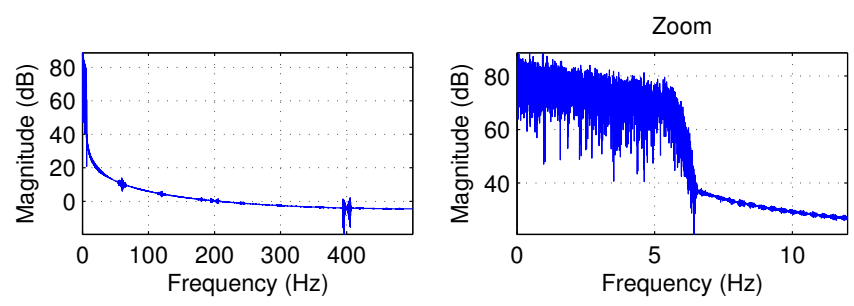

(a)
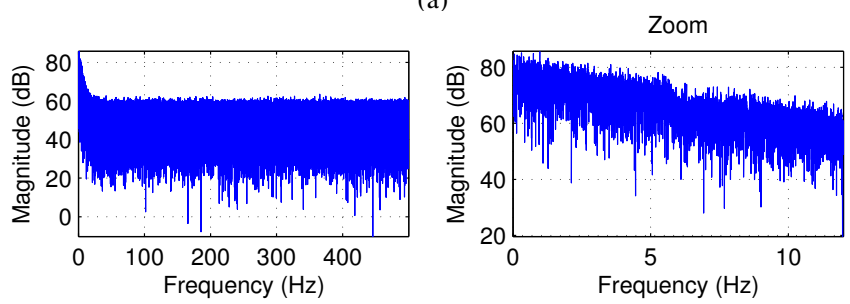

(b)
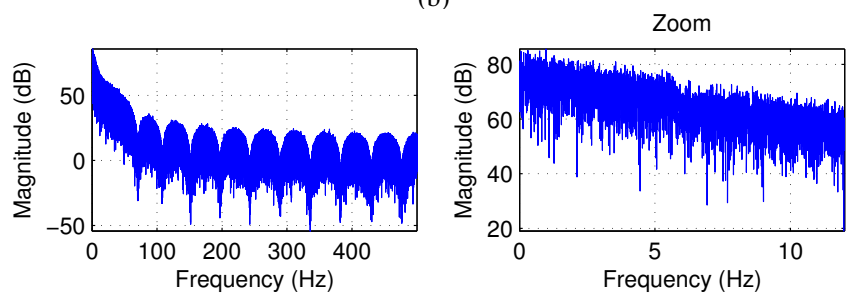

(c)

Fig. 5. Frequency domain analysis (magnitude), $S N R=6 \mathrm{~dB}, f_{d}=6 \mathrm{~Hz}$. The figures in the right panes are a zoom of the frequency. (a) $H_{t}[k]$; (b) $\widehat{H}_{t}[k]$; (c) Filtered $\widehat{H}_{t}[k]$.

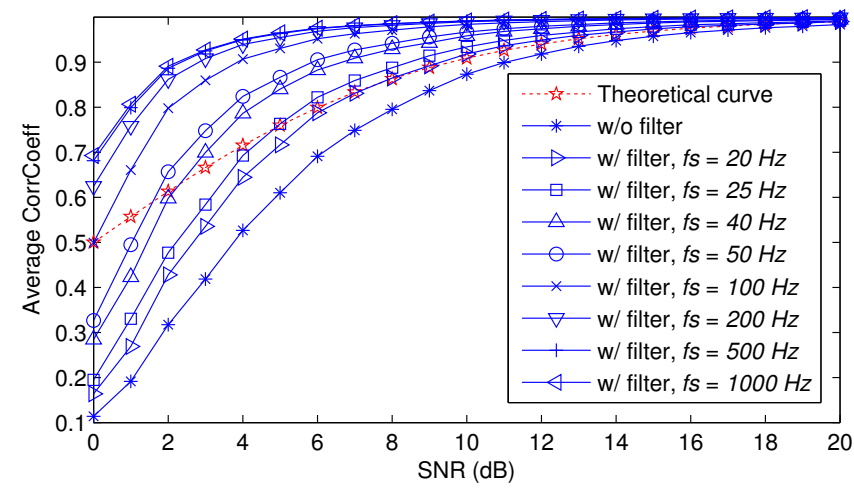

Fig. 6. The average correlation coefficient in different SNR environments under an LPF with different sampling frequency.

energy of the $H_{t}[k]$ is in the range of $\left[0, f_{d}\right]$, an LPF with a cutoff frequency $f_{c}$ of $f_{d}$ is designed to target elimination of the high frequency components of the noise. However, the estimation of the Doppler spread is difficult, thus $f_{c}$ is fixed to $f_{d, \max }$. Key generation has been conventionally aimed at slow fading environments so that $f_{d, \max }$ is very small, e.g., $6 \mathrm{~Hz}$ in a Bell-shaped Doppler power spectrum model [42]. Therefore, keeping $f_{c}$ to $f_{d, \max }$ fixed will not greatly impact the performance.

The noise suppression effect of the LPF is shown in Fig. 5 (c); it may be observed that the high frequency components of the noise is largely eliminated. The improvement of the correlation relationship for all the subcarriers when
TABLE II

PARAMETERS OF THE DESIGNED LPF

\begin{tabular}{|l|l|}
\hline Cutoff frequency $f_{c}$ & $f_{d, \max }$ \\
\hline Filter order & 20 \\
\hline Kaiser window length & 21 \\
\hline Kaiser window $\beta$ & 3 \\
\hline
\end{tabular}

$S N R=6 \mathrm{~dB}$ is shown in Fig. 3, from which it may be observed that all the subcarriers have quite similar correlation coefficients after filtering.

The performance of the LPF with varying sampling frequencies $f_{s}$ in different SNR environments is shown in Fig. 6. It may be observed from the figure that the LPF produces a good improvement of the correlation, especially in low SNR environments. Ideally, a higher sampling frequency $f_{s}$ is preferred due to its better improvement. However, when the channel changes slowly and the sampling frequency reaches some value, e.g., $200 \mathrm{~Hz}$ in Fig. 6, any further increase in the sampling frequency does not contribute much more to the sampling of the signal variation. Therefore, it is not necessary to use a very high sampling frequency because an optimal sampling frequency can be tuned to the signal variation. This could benefit the application of LPF in cost- and energy-sensitive devices as it can keep the overhead introduced by LPF as low as possible while achieving an acceptable performance.

The hardware cost for the filter is low as it has a small order. In addition, current $3 \mathrm{G}$ cellular devices regularly monitor the channel at $1500 \mathrm{~Hz}$ for closed loop power control. As may be observed from Fig. 6, a sampling frequency of $200 \mathrm{~Hz}$ already produces a good improvement on the correlation relationship. Hence, the sampling overhead is well within the capability of mobile devices. Therefore, the implementation of the LPF is worthwhile to improve the cross-correlation of the measurements, while introducing only a small overhead and cost.

\section{Performance Evaluation}

The channel responses of OFDM subcarriers are sampled at a frequency $f_{s}$. The sampled data $\widehat{H}_{q}[k]$ is then passed to the LPF in order to improve the cross-correlation relationship. The filter data is later re-sampled by a probing rate $T_{p}$ to reduce the redundancy. In our system, a single-bit cumulative distribution function (CDF)-based quantization [16] is adopted to convert $\widehat{H}_{q}[k]$ into binary values $K_{k}$. These binary sequences may be used separately as keys to different cryptographic applications. Alternatively, we can concatenate multiple binary sequences together to form a longer sequence, i.e., $K=$ $\left[K_{1}\|\ldots\| K_{k} \| \ldots|| K_{N_{s}}\right]$, where $\|$ denotes concatenation and $N_{s}$ is the number of uncorrelated subcarriers, which will be analyzed in detail in Section V-A2. Information reconciliation technique, such as secure sketch [46], is used to correct the key disagreement between the users, and privacy amplification is finally employed to remove the information revealed to eavesdroppers during the information reconciliation.

In this section, we evaluated the performance of our key generation system in terms of randomness, KGR, and KDR. 
TABLE III

RANDOMNESS TEST RESULTS OF KEY SEQUENCES QUANTIZED FROM $\widehat{H}_{q}[k]$. THE PROBING RATES $T_{p}$ ARE SET AS DIFFERENT $X \%$ COHERENCE TIME $T_{c}(X \%)$.

\begin{tabular}{|l|l|l|l|l|l|l|}
\hline Corr coeff $X \%$ & $50 \%$ & $30 \%$ & $15 \%$ & $12 \%$ & $10 \%$ & $9 \%$ \\
\hline$T_{c}(X \%)(\mathrm{s})$ & 0.067 & 0.097 & 0.136 & 0.154 & 0.2 & 0.226 \\
\hline Sequence length & 7462 & 5154 & 3676 & 3246 & 2500 & 2212 \\
\hline Frequency & 0.61 & 0.956 & 0.767 & 0.861 & 0.968 & 0.799 \\
\hline Block frequency & 0 & 0.001 & 0.242 & 0.185 & 0.408 & 0.021 \\
\hline Runs & 0 & 0 & 0 & 0.001 & 0.02 & 0.046 \\
\hline Longest run of 1s & 0 & 0 & 0.014 & 0.824 & 0.85 & 0.668 \\
\hline DFT & 0 & 0.005 & 0.283 & 0.729 & 0.054 & 0.654 \\
\hline Serial & 0 & 0 & 0.21 & 0.104 & 0.211 & 0.495 \\
& 0 & 0.257 & 0.943 & 0.88 & 0.107 & 0.667 \\
\hline Appro. entropy & 0 & 0 & 0 & 0.012 & 0.282 & 0.472 \\
\hline Cum. sums (fwd) & 0.521 & 0.652 & 0.565 & 0.854 & 0.967 & 0.252 \\
\hline Cum. sums (rev) & 0.316 & 0.704 & 0.837 & 0.743 & 0.981 & 0.404 \\
\hline
\end{tabular}

TABLE IV

POWER DISTRIBUTION OF CIR UNDER EXPONENTIAL-DECAY POWER DELAY PROFILE. THE TOTAL POWER $\sum_{l=0}^{L-1} \sigma_{h_{l}}^{2}$ IS NORMALIZED TO 1.

\begin{tabular}{|l|l|l|l|l|}
\hline$L$ & $\sigma_{h_{1}}^{2}$ & $\sum_{l=0}^{1} \sigma_{h_{l}}^{2}$ & $\sum_{l=0}^{2} \sigma_{h_{l}}^{2}$ & $\sum_{l=0}^{3} \sigma_{h_{l}}^{2}$ \\
\hline 6 & $86.47 \%$ & $98.17 \%$ & $99.75 \%$ & $99.96 \%$ \\
\hline 11 & $63.21 \%$ & $86.46 \%$ & $95.01 \%$ & $98.16 \%$ \\
\hline 21 & $39.35 \%$ & $63.21 \%$ & $77.69 \%$ & $86.47 \%$ \\
\hline
\end{tabular}

\section{A. Randomness Test}

1) Single Random Source: A statistical randomness test suite provided by National Institute of Standards and Technology (NIST) [47] is adopted to test the randomness of the key sequence generated from the channel responses of OFDM subcarriers, which is widely used in the key generation systems [12], [13], [20], [22]. Table III shows the results of the randomness test of keys quantized from a single subcarrier. Each test returns a $P$-value which is compared with a threshold (0.01 in this paper). The cells highlighted in gray fail the random test, i.e., $P$-value $<0.01$.

As may be observed from the Table III, using the commonly acknowledged $50 \%$ coherence time $T_{c}(50 \%)$ as the probing rate cannot generate random sequences at all. In these results, the probing rate needs to be increased to $T_{c}(10 \%)$ in order for the system to be able to extract a random key sequence. This is the optimal probing rate.

Temporal correlation can also be tackled by using decorrelation algorithms [16], [25]. The decorrelation algorithms themselves do not introduce more entropy but only aggregate the energy. In addition, the algorithms' complexities increase with the data block length [48], which may not be applicable to limited computational capacity devices. A rule of thumb for the optimal probing rate is thus attractive as it does not require any other additional signal processing.

2) Multiple Random Source: In a multipath channel with $L$ independent channel taps, theoretically there should be up to $L$ independent subcarriers. However, the average power of the taps is not evenly distributed. For example, it follows an exponential-decay profile in the indoor environment and the power will be mostly concentrated in the first few taps, as shown in Table IV.

Only the taps with short delays are the main contributors to the randomness. Therefore, the number of uncorrelated subcarriers for key generation $N_{s}$ will also be smaller than $L$. In this section, we selected $N_{s}$ subcarriers satisfying

$$
-0.5<R_{\widehat{H}}\left(f_{k}, f_{i}, 0\right)<0.5,
$$

quantized them separately and finally concatenated these binary values to form a new sequence. As may be observed from Fig. 1, subcarriers have slightly different $T_{c}(X \%)$. In order to focus on the frequency correlation between two binary sequences $K_{k}$ and $K_{i}$, we use a relatively large probing rate, $0.5 \mathrm{~s}$, so there will be little temporal correlation within $K_{k}$.

NIST randomness test is applied to the new sequence and the results are shown in Table V. We also did the same process to the theoretical channel response $H_{q}[k]$ for comparison. For all the multipath environments, $N_{s}<L$, which matches our intuitive analysis that the first $N_{s}$ channel taps are the dominant contributor to the randomness. In addition, when there is richer scattering in the environment, i.e., more channel taps, there are more random sources for extraction, which is due to that the channel is more frequency-selective.

\section{B. $K G R$}

Channel parameter (CSI, RSS, etc) and probing rate are the key factors for the KGR. In this paper, due to the employment of the fine-grained channel responses of OFDM subcarriers and determination of optimal probing rate, our system can achieve a much higher KGR than existing single-dimensional parameter-based key generation systems.

The KGR of single-dimensional parameter-based key generation systems, e.g., RSS-based systems, can be written as

$$
K G R^{\prime}=\frac{1}{T_{p}} .
$$

Single-dimensional parameter-based key generation systems lose lots of useful information of the channel. For example, RSS only has amplitude information.

Our scheme can achieve a higher KGR than singledimensional parameter-based schemes. Firstly, we can extract keys from the real and imaginary parts of the channel estimation simultaneously, a general feature of key generation from fine-grained CSI [25], [49], which can double the KGR compared to the single-dimensional parameter-based systems. Secondly, we extract randomness from both the time and frequency domains. In particular, in a frequency-selective fading channel, there are up to $N_{s}$ frequencies applicable for key generation in our scheme, which will significantly improve the KGR. Therefore, the KGR of our system can be given as

$$
K G R=\sum_{i=1}^{N_{s}} \frac{2}{T_{p}(i)},
$$

where $T_{p}(i)$ is $i^{t h}$ subcarrier's optimal probing rate.

\section{C. $K D R$}

As can be observed from Fig. 7, even with the help of the LPF, there is still disagreement between Alice and Bob. This is because the noise effect can only be suppressed, but not 
TABLE V

RANDOMNESS TEST RESULTS OF KEY SEQUENCES CONCATENATED FROM MULTIPLE SUBCARRIERS

\begin{tabular}{|l|l|l|l|l|l|l|l|l|l|l|l|l|l|}
\hline$L$ & \multicolumn{4}{|c|}{6} & \multicolumn{3}{|c|}{11} & \multicolumn{3}{c|}{21} \\
\hline Data & \multicolumn{2}{|c|}{$H_{q}[k]$} & \multicolumn{2}{|c|}{$\widehat{H}_{q}[k]$} & \multicolumn{2}{|c|}{$H_{q}[k]$} & \multicolumn{3}{c|}{$\widehat{H}_{q}[k]$} & \multicolumn{3}{|c|}{$H_{q}[k]$} & $\widehat{H}_{q}[k]$ \\
\hline$N_{s}$ & 1 & 2 & 3 & 4 & 3 & 4 & 4 & 5 & 8 & 9 & 9 & 10 \\
\hline Sequence length & 1000 & 2000 & 3000 & 4000 & 3000 & 4000 & 4000 & 5000 & 8000 & 9000 & 9000 & 10000 \\
\hline \hline Frequency & 0.899 & 0.928 & 1 & 0.635 & 0.97 & 0.949 & 0.949 & 0.909 & 0.84 & 0.899 & 0.966 & 0.984 \\
\hline Block frequency & 0.958 & 0.604 & 0.393 & 0.245 & 0.477 & 0.941 & 0.939 & 0.478 & 0.987 & 0.942 & 0.282 & 0.417 \\
\hline Runs & 0.164 & 0.152 & 0.77 & 0.129 & 0.798 & 0.429 & 0.974 & 0.887 & 0.035 & 0.016 & 0.849 & 0.968 \\
\hline Longest run of 1s & 0.66 & 0.164 & 0.522 & 0.712 & 0.953 & 0.688 & 0.824 & 0.962 & 0.286 & 0.024 & 0.518 & 0.651 \\
\hline DFT & 0.384 & 0 & 0.093 & 0 & 0.019 & 0 & 0.146 & 0 & 0.01 & 0 & 0.033 & 0 \\
\hline Serial & 0.423 & 0.101 & 0.928 & 0.663 & 0.237 & 0.771 & 0.199 & 0.336 & 0.324 & 0.528 & 0.594 & 0.769 \\
& 0.332 & 0.041 & 0.841 & 0.754 & 0.454 & 0.8 & 0.1 & 0.328 & 0.623 & 0.137 & 0.636 & 0.664 \\
\hline Appro. entropy & 0.33 & 0.254 & 0.988 & 0.228 & 0.305 & 0.809 & 0.692 & 0.398 & 0.044 & 0.511 & 0.367 & 0.591 \\
\hline Cum sum (fwd) & 0.989 & 0.956 & 0.948 & 0.736 & 0.744 & 0.965 & 0.989 & 0.954 & 0.997 & 0.999 & 0.979 & 0.986 \\
\hline Cum sum (rev) & 0.999 & 0.902 & 0.948 & 0.876 & 0.778 & 0.986 & 0.989 & 0.881 & 0.999 & 0.987 & 0.963 & 0.981 \\
\hline
\end{tabular}

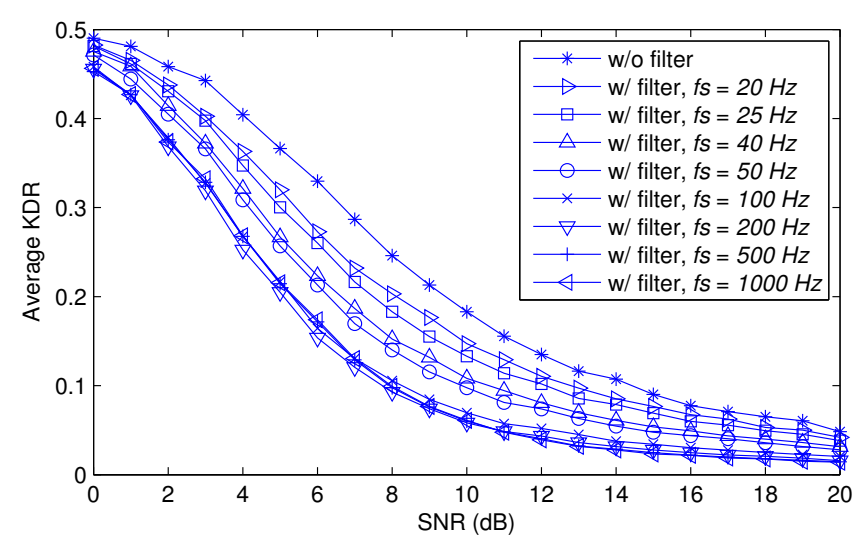

Fig. 7. The average KDR in different SNR environments

completely eliminated. Therefore, information reconciliation is necessary to make Alice and Bob agree on the same key. However, all the information reconciliation techniques are upper bounded by the correction capacity. Taking the secure sketch [46] as an example, the $[n, k, t] \mathrm{BCH}$ code can be implemented to correct the disagreement with a maximum correction capacity rate of

$$
\eta=\frac{t_{\max }}{n}=\frac{2^{m-2}-1}{2^{m}-1}
$$

which approaches 0.25 when $m$ becomes large. The KDR should be smaller than the correction capacity $\eta$ in order to guarantee all the disagreement to be corrected by information reconciliation. There is a lower bound of SNR for the key generation working successfully, which equals $8 \mathrm{~dB}$ when there is no LPF, or $4 \mathrm{~dB}$ when the correlation is improved by the LPF with sampling frequency $f_{s}=100 \mathrm{~Hz}$ or higher, as shown in Fig. 7. This extends the working SNR range by $4 \mathrm{~dB}$. Even in high SNR environments, the introduction of LPF is still beneficial. A reduction in the KDR decreases the burden of the information reconciliation, and can ease its design. In addition, a lower KDR requires fewer rounds of information reconciliation and less information is revealed to eavesdroppers. Therefore, the correlation improvement by LPF can make the key generation system much more efficient.

\section{CONCLusion And Future Work}

An efficient key generation system that exploits the randomness from OFDM subcarrier's channel responses is proposed. The efficiency is achieved by using an optimal probing rate, randomness extraction from multiple subcarriers, and improved cross-correlation of the measurements.

The appropriateness of OFDM subcarrier's channel responses as a random source for key generation is verified through theoretical modelling and analysis. The time and frequency autocorrelation relationship of the OFDM subcarrier's channel responses is modelled theoretically and it helps determine the optimal probing rate and the number of subcarriers that can be used for key extraction. Crosscorrelation of the channel measurements is modelled and noise is found to have a more detrimental effect than nonsimultaneous measurements in a slow fading channel. An LPF is subsequently proposed to suppress the high frequency components of noise, improve the cross-correlation coefficient and reduce the KDR, which extends the SNR working range of the system. We evaluated our system in terms of randomness, KGR and KDR, and showed that OFDM subcarrier's channel responses are valid for key generation. In a real environment, the channel may change dynamically due to uncontrolled movement of users/objects, which results in variable statistical channel features, such as varying Doppler spread and coherence bandwidth. Optimal probing rate and uncorrelated subcarriers selection are determined by Doppler spread and coherence bandwidth, respectively. Our future work will be to design an adaptive key generation system exploiting randomness from time and frequency domains, which adjusts the probing parameters according to the channel condition.

\section{REFERENCES}

[1] A. Attar, H. Tang, A. V. Vasilakos, F. R. Yu, and V. Leung, "A survey of security challenges in cognitive radio networks: Solutions and future research directions," Proc. IEEE, vol. 100, no. 12, pp. 3172-3186, 2012.

[2] H. Yang, Y. Zhang, Y. Zhou, X. Fu, H. Liu, and A. V. Vasilakos, "Provably secure three-party authenticated key agreement protocol using smart cards," Computer Networks, vol. 58, pp. 29-38, 2014.

[3] J. Zhou, X. Dong, Z.-F. Cao, and A. Vasilakos, "Secure and privacy preserving protocol for cloud-based vehicular DTNs," IEEE Trans. Inf. Forensics Security, vol. 10, no. 6, pp. 1299-1314, 2015.

[4] Y. Zou, X. Wang, and L. Hanzo, "A survey on wireless security: technical challenges, recent advances and future trends," pp. 1-31, 2015. [Online]. Available: http://arxiv.org/abs/1505.07919 
[5] Z. Zhang, X. Chai, K. Long, A. V. Vasilakos, and L. Hanzo, "Full duplex techniques for 5G networks: Self-interference cancellation, protocol design, and relay selection," IEEE Commun. Mag., vol. 53, no. 5, pp. 128-137, 2015.

[6] Z. Zhang, X. Wang, K. Long, A. V. Vasilakos, and L. Hanzo, "Largescale MIMO-based wireless backhaul in 5G networks," IEEE Wireless Commun. Mag., vol. 22, no. 5, pp. 58-66, 2015.

[7] A. Mukherjee, S. Fakoorian, J. Huang, and A. Swindlehurst, "Principles of physical layer security in multiuser wireless networks: A survey," IEEE Commun. Surveys Tuts., vol. 16, no. 3, pp. 1550-1573, 2014.

[8] T. Wang, Y. Liu, and A. V. Vasilakos, "Survey on channel reciprocity based key establishment techniques for wireless systems," Wireless Networks, pp. 1-12, Jan. 2015.

[9] J. Zhang, T. Q. Duong, A. Marshall, and R. Woods, "Key generation from wireless channels: A review," IEEE Access, vol. 4, pp. 614-626, 2016.

[10] R. Ahlswede and I. Csiszar, "Common randomness in information theory and cryptography - Part I: secret sharing," IEEE Trans. Inf. Theory, vol. 39, no. 4, pp. 1121-1132, 1993.

[11] S. Ali, V. Sivaraman, and D. Ostry, "Eliminating reconciliation cost in secret key generation for body-worn health monitoring devices," IEEE Trans. Mobile Comput., vol. 13, no. 12, pp. 2763-2776, 2014.

[12] S. Mathur, W. Trappe, N. Mandayam, C. Ye, and A. Reznik, "Radiotelepathy: Extracting a secret key from an unauthenticated wireless channel," in Proc. 14th Annu. Int. Conf. on Mobile Computing and Networking (MobiCom), San Francisco, CA, USA, Sep. 2008, pp. 128 139.

[13] S. Jana, S. N. Premnath, M. Clark, S. K. Kasera, N. Patwari, and S. V. Krishnamurthy, "On the effectiveness of secret key extraction from wireless signal strength in real environments," in Proc. 15th Annu. Int. Conf. on Mobile Computing and Networking (MobiCom), Beijing, China, Sep. 2009, pp. 321-332.

[14] K. Zeng, D. Wu, A. Chan, and P. Mohapatra, "Exploiting multipleantenna diversity for shared secret key generation in wireless networks," in Proc. 29th IEEE Int. Conf. on Computer Communications (INFOCOM), San Diego, California, USA, Mar. 2010, pp. 1-9.

[15] H. Liu, J. Yang, Y. Wang, and Y. Chen, "Collaborative secret key extraction leveraging received signal strength in mobile wireless networks," in Proc. 31st IEEE Int. Conf. on Computer Communications (INFOCOM), Orlando, Florida, USA, Mar. 2012, pp. 927-935.

[16] N. Patwari, J. Croft, S. Jana, and S. K. Kasera, "High-rate uncorrelated bit extraction for shared secret key generation from channel measurements," IEEE Trans. Mobile Comput., vol. 9, no. 1, pp. 17-30, 2010.

[17] L. Yao, S. T. Ali, V. Sivaraman, and D. Ostry, "Decorrelating secret bit extraction via channel hopping in body area networks," in Proc. 23rd IEEE Int. Symp. on Personal Indoor and Mobile Radio Communications (PIMRC), Sydney, Australia, Sep. 2012, pp. 1454-1459.

[18] M. Wilhelm, I. Martinovic, and J. B. Schmitt, "Secure key generation in sensor networks based on frequency-selective channels," IEEE J. Sel. Areas Commun., vol. 31, no. 9, pp. 1779-1790, 2013.

[19] Q. Wang, H. Su, K. Ren, and K. Kim, "Fast and scalable secret key generation exploiting channel phase randomness in wireless networks," in Proc. 30th IEEE Int. Conf. on Computer Communications (INFOCOM), Shanghai, China, Apr. 2011, pp. 1422-1430.

[20] Q. Wang, K. Xu, and K. Ren, "Cooperative secret key generation from phase estimation in narrowband fading channels," IEEE J. Sel. Areas Commun., vol. 30, no. 9, pp. 1666-1674, 2012.

[21] B. Azimi-Sadjadi, A. Kiayias, A. Mercado, and B. Yener, "Robust key generation from signal envelopes in wireless networks," in Proc. 14th ACM Conf. on Computer and Communications Security (CCS), Alexandria, USA, Oct. 2007, pp. 401-410.

[22] H. Liu, Y. Wang, J. Yang, and Y. Chen, "Fast and practical secret key extraction by exploiting channel response," in Proc. 32nd IEEE Int. Conf. on Computer Communications (INFOCOM), Turin, Italy, Apr. 2013, pp. 3048-3056.

[23] W. Xi, X. Li, C. Qian, J. Han, S. Tang, J. Zhao, and K. Zhao, "KEEP: Fast secret key extraction protocol for D2D communication," in Proc. 22nd IEEE Int. Symp. of Quality of Service (IWQoS), Hong Kong, May 2014, pp. 350-359.

[24] J. W. Wallace and R. K. Sharma, "Automatic secret keys from reciprocal MIMO wireless channels: Measurement and analysis," IEEE Trans. Inf. Forensics Security, vol. 5, no. 3, pp. 381-392, 2010.

[25] C. Chen and M. A. Jensen, "Secret key establishment using temporally and spatially correlated wireless channel coefficients," IEEE Trans. Mobile Comput., vol. 10, no. 2, pp. 205-215, 2011
[26] B. Quist and M. Jensen, "Maximizing the secret key rate for informed radios under different channel conditions," IEEE Trans. Wireless Commun., vol. 12, no. 10, pp. 5146-5153, Oct. 2013.

[27] E. A. Jorswieck, A. Wolf, and S. Engelmann, "Secret key generation from reciprocal spatially correlated MIMO channels," in Proc. IEEE GLOBECOM Workshop on Trusted Communications with Physical Layer Security (TCPLS), Atlanta, GA USA, Dec. 2013, pp. 1245-1250.

[28] B. Quist and M. Jensen, "Maximization of the channel-based key establishment rate in MIMO systems," IEEE Trans. Wireless Commun., vol. 14 , no. 10 , pp. 5565 - 5573, 2015.

[29] R. Wilson, D. Tse, and R. Scholtz, "Channel identification: Secret sharing using reciprocity in ultrawideband channels," IEEE Trans. Inf. Forensics Security, vol. 2, no. 3, pp. 364-375, 2007.

[30] S. Mathur, R. Miller, A. Varshavsky, W. Trappe, and N. Mandayam, "Proximate: Proximity-based secure pairing using ambient wireless signals," in Proc. 9th Int. Conf. on Mobile Systems, Applications, and Services (MobiSys), Washington, DC, USA, Jul. 2011, pp. 211-224.

[31] X. Zhu, F. Xu, E. Novak, C. C. Tan, Q. Li, and G. Chen, "Extracting secret key from wireless link dynamics in vehicular environments," in Proc. 32nd IEEE Int. Conf. on Computer Communications (INFOCOM), Turin, Italy, Apr. 2013, pp. 2283-2291.

[32] WiPhyLoc8 Project. [Online]. Available: http://wiphyloc8.org/

[33] J. Zhang, A. Marshall, R. Woods, and T. Q. Duong, "Secure key generation from OFDM subcarriers' channel responses," in Proc. IEEE GLOBECOM Workshop on Trusted Communications with Physical Layer Security (TCPLS), Austin, USA, Dec. 2014, pp. 1302-1307.

[34] J. Zhang, R. Woods, A. Marshall, and T. Q. Duong, "An effective key generation system using improved channel reciprocity," in Proc. 40th IEEE Int. Conf. on Acoustics, Speech and Signal Processing (ICASSP), Brisbane, Australia, Apr. 2015, pp. 1727-1731.

[35] P. Bello, "Characterization of randomly time-variant linear channels," IEEE Trans. on Commun. Systems, vol. 11, no. 4, pp. 360-393, 1963.

[36] J. Wallace, M. Jensen, B. D. Jeffs et al., "Experimental characterization of the MIMO wireless channel: Data acquisition and analysis," IEEE Trans. Wireless Commun., vol. 2, no. 2, pp. 335-343, 2003.

[37] A. F. Molisch, K. Balakrishnan, D. Cassioli, C.-C. Chong, S. Emami, A. Fort, J. Karedal, J. Kunisch, H. Schantz, U. Schuster et al., "IEEE 802.15.4a channel model-final report," IEEE, Tech. Rep. 802.15 04/662r0, 2004.

[38] H. Minn, V. K. Bhargava, and K. B. Letaief, "A robust timing and frequency synchronization for OFDM systems," IEEE Trans. Wireless Commun., vol. 2, no. 4, pp. 822-839, 2003.

[39] Y. S. Cho, J. Kim, W. Y. Yang, and C. G. Kang, MIMO-OFDM Wireless Communications with MATLAB. Wiley-IEEE Press, 2010.

[40] Wireless LAN Medium Access Control (MAC) and Physical Layer (PHY) Specification, IEEE Std. 802.11, 2012.

[41] C.-D. Iskander, "A MATLAB-based object-oriented approach to multipath fading channel simulation," Mathworks, Natick, MA, White Paper 18869, Feb. 2008

[42] V. Erceg et al., "TGn channel models," IEEE, Tech. Rep. 802.11 03/940r4, May 2004.

[43] Y. Li, L. J. Cimini, and N. R. Sollenberger, "Robust channel estimation for OFDM systems with rapid dispersive fading channels," IEEE Trans. Commun., vol. 46, no. 7, pp. 902-915, 1998.

[44] T. S. Rappaport, Wireless Communications: Principles and Practice, 2nd ed. Prentice Hall, 2001.

[45] H. Jung, T. Kwon, K. Cho, and Y. Choi, "REACT: Rate adaptation using coherence time in 802.11 WLANs," Computer Communications, vol. 34, no. 11 , pp. 1316-1327, 2011.

[46] Y. Dodis, R. Ostrovsky, L. Reyzin, and A. Smith, "Fuzzy extractors: How to generate strong keys from biometrics and other noisy data," SIAM J. on Computing, vol. 38, no. 1, pp. 97-139, 2008.

[47] A. Rukhin et al., "A statistical test suite for random and pseudorandom number generators for cryptographic applications," National Institute of Standards and Technology, Tech. Rep. Special Publication 800-22 Revision 1a, Apr. 2010.

[48] S. Gopinath, R. Guillaume, P. Duplys, and A. Czylwik, "Reciprocity enhancement and decorrelation schemes for PHY-based key generation," in Proc. IEEE GLOBECOM Workshop on Trusted Communications with Physical Layer Security (TCPLS), Austin, USA, Dec. 2014, pp. 13671372.

[49] J. W. Wallace, C. Chen, M. Jensen et al., "Key generation exploiting MIMO channel evolution: Algorithms and theoretical limits," in Proc. 3rd European Conf. Antennas and Propagation (EuCAP), Berlin, Germany, Mar. 2009, pp. 1499-1503. 